\title{
Prospective surveillance model in the home for breast cancer-related lymphoedema: a feasibility study
}

\author{
Louise A. Koelmeyer ${ }^{1}$ (D) Emma Moloney ${ }^{1} \cdot$ John Boyages $^{1,2} \cdot$ Kerry A. Sherman $^{3} \cdot$ Catherine M. Dean $^{4}$
}

Received: 13 August 2020 / Accepted: 23 September 2020 / Published online: 1 October 2020

๑) Springer Science+Business Media, LLC, part of Springer Nature 2020

\begin{abstract}
Purpose The aim of this study was to assess the feasibility of delivering a prospective surveillance model in the home over 6 months for women at high risk of developing lymphoedema.

Methods A single-group, intervention study recruited 20 women who had surgical and medical treatment for confirmed node-positive invasive breast cancer and therefore at high risk of developing arm lymphoedema. Participants received a package including Bioimpedance Spectroscopy (BIS) monitoring, lymphoedema education and support to promote selfmanagement and physical activity.

Results Participants adhered to BIS monitoring $74 \%$ of the time, and felt extremely confident in using the device. By 6 months, mean BIS L-Dex scores had increased from 3.5 (SD 5.6) to 8.4 (SD 11.1); five women (25\%) who experienced $>+6.5$ increase in L-Dex score were fitted with a compression garment. Self-reported symptoms and distress decreased by 0.4 out of 10 (95\% CI 0.1 to 0.7 ); number of self-management strategies used increased by 0.6 (95\% CI 0.1 to 1.2 ); and planned exercise increased by $2.8 \mathrm{~h} /$ week (95\% CI 0.4 to 5.2 ).

Conclusions These findings indicate a prospective surveillance model of care in the home with BIS is feasible and associated with increased self-management. A Phase II randomised trial is warranted as well as research exploring the costs associated with implementing this model of care for high-risk individuals.
\end{abstract}

Keywords Breast cancer-related lymphoedema $\cdot$ Bioimpedance spectroscopy (BIS) $\cdot$ Home monitoring $\cdot$ Prospective surveillance model $\cdot$ Phase I trial $\cdot$ Feasibility

\section{Introduction}

Breast cancer-related lymphoedema is an important survivorship issue for individuals completing treatment for breast cancer because it reduces quality of life [1-4] and may cause

Louise A. Koelmeyer

Louise.koelmeyer@mq.edu.au

1 Australian Lymphoedema Education, Research and Treatment (ALERT) Program, Department of Clinical Medicine, Faculty of Medicine, Health and Human Sciences, Macquarie University, Sydney, Australia

2 Icon Cancer Centre, Sydney, NSW, Australia

3 Centre for Emotional Health, Department of Psychology, Faculty of Medicine, Health and Human Sciences, Macquarie University, Sydney, Australia

4 Department of Health Professions, Faculty of Medicine, Health and Human Sciences, Macquarie University, Sydney, Australia physical, psychological and financial impairments [1, 5-10]. The incidence of lymphoedema following breast cancer treatment varies and ranges from 4 to $47 \%$ depending on measurement tool used, lymphoedema diagnosis criteria, as well as patient and treatment-related factors [11-13].

A prospective surveillance and early intervention model of care has been recognised as an optimal framework for the early detection and management of sub-clinical lymphoedema [13-15]. Using a prospective surveillance model in clinical practice enables the early identification and intervention for lymphoedema, sometimes in a sub-clinical stage, where intervention can prevent progression to a chronic condition [16-19]. Recommended practice for the prospective surveillance model requires that individuals at risk of developing lymphoedema regularly attend clinics on a 3-6 monthly cycle for 3 years as this is the most common timeframe for detecting sub-clinical lymphoedema $[13,16]$. Developments in technology for monitoring lymphoedema allow for the identification of sub-clinical lymphoedema 
using bioimpedance spectroscopy (BIS) $[12,20,21]$ that utilises an "impedance ratio" to assess unilateral lymphoedema of the arm [22].

The ability to provide regular feedback in real time has been shown to enhance the effectiveness of immediate and ongoing lymphoedema self-care [23]. Women have expressed that self-monitoring and management within the home may be beneficial during a challenging period of their life coping with cancer [24]. BIS technology is quick and easy to use, and therefore, has the potential to be used for 'home monitoring' of sub-clinical lymphoedema in the intervals between formal monitoring in the clinic. Up until now, individuals at high risk of developing lymphoedema have had no means by which to objectively monitor their symptoms, relying instead on subjective feelings of swelling, pain and discomfort $[25,26]$. A newer, more user-friendly stand-on device becoming available now enables it to be tested within the home. It is anticipated that if individuals can monitor themselves at home for early changes in extracellular fluid, then they may be empowered to access earlier intervention which may improve long-term quality of life [24].

The primary aim of this study was to assess the feasibility and potential benefit of a prospective surveillance model in the home for 6 months for women at high risk of developing lymphoedema (i.e. confirmed node-positive invasive breast cancer after undergoing an axillary lymph node dissection or sentinel lymph node biopsy). While lymphoedema may emerge over a 3-year period, for this feasibility study we selected a surveillance period of 6 months because it was double the duration of the only previous home monitoring pilot study and the stand-on BIS device had not been used in the home [23]. The specific research questions were as follows:

(1) Is it feasible (in terms of recruitment, adherence, confidence, usefulness, technology and measurement) to deliver a prospective surveillance model in the home for 6 months for women at risk of developing lymphoedema?

(2) Does a prospective surveillance model in the home for 6 months have the potential to decrease lymphoedema, symptom intensity and distress, increase lymphoedema self-management, and physical activity in the shortterm (3 months) and in the longer term (6 months)?

\section{Method}

\section{Design}

A Phase I, single-group, intervention study was undertaken (Fig. 1). Women who had undergone surgical and medical treatment for breast cancer and were at high risk for developing unilateral arm lymphoedema were recruited from three multidisciplinary breast cancer clinics within northern suburbs in Sydney, Australia. Women were initially screened via the telephone by research assistant and eligible participants were scheduled for a baseline assessment in their home 3 months after breast cancer surgery. Measurements were taken at baseline ( 3 months post breast surgery), 3 and 6 months in the home by trained measurers following standardised protocols. The intervention consisting of education and support components of the model of care were provided at the conclusion of each measurement session. This study was approved by Macquarie University Human Research Ethics Committee (Reference number 5201800197) and the Adventist HealthCare Limited Human Research Ethics Committee (Reference number 2018-036).

\section{Participants}

Women who were aged between 18 and 85 years with a histologically confirmed node-positive invasive breast cancer after undergoing an axillary lymph node dissection or sentinel lymph node biopsy, were able to speak and read English, and were capable of giving informed written consent were invited to participate in the study. Individuals were excluded if they had implantable devices, such as a pacemaker or other inbuilt stimulator; were pregnant; reported having a previous history of breast cancer or arm lymphoedema; had a health condition that may affect body fluid status; or reported having a psychiatric illness that would limit their compliance with study requirements.

\section{Intervention}

Participants received the prospective surveillance model of care in the home for 6 months. This consisted of a package including BIS monitoring, education and support to promote self-management and physical activity. The education component focussed on risk minimisation and included provision of the Australian Cancer Council Understanding Lymphoedema fact sheet [27]. Self-management strategies included skin care, exercise, self-lymphatic drainage massage, compression and the use of pneumatic compression.

BIS monitoring involved the use of a BIS stand-on device. The stand-on device used was a commercially available impedance device $\left(\mathrm{SOZO}^{\circledR}\right.$, ImpediMed Limited, Brisbane, Australia) with demonstrated validity and reliability [28]. BIS measures the resistance at $0 \mathrm{kHz}$ (R0) of the unaffected limb and compares this to the resistance at $0 \mathrm{kHz}$ of the affected/at-risk limb expressed as the following ratio (unaffected:affected/at-risk). Alternatively, this ratio may be linearised and expressed as an L-Dex score with the normal range being between -10 and +10 [22]. A 
Fig. 1 Flow of participants to the study

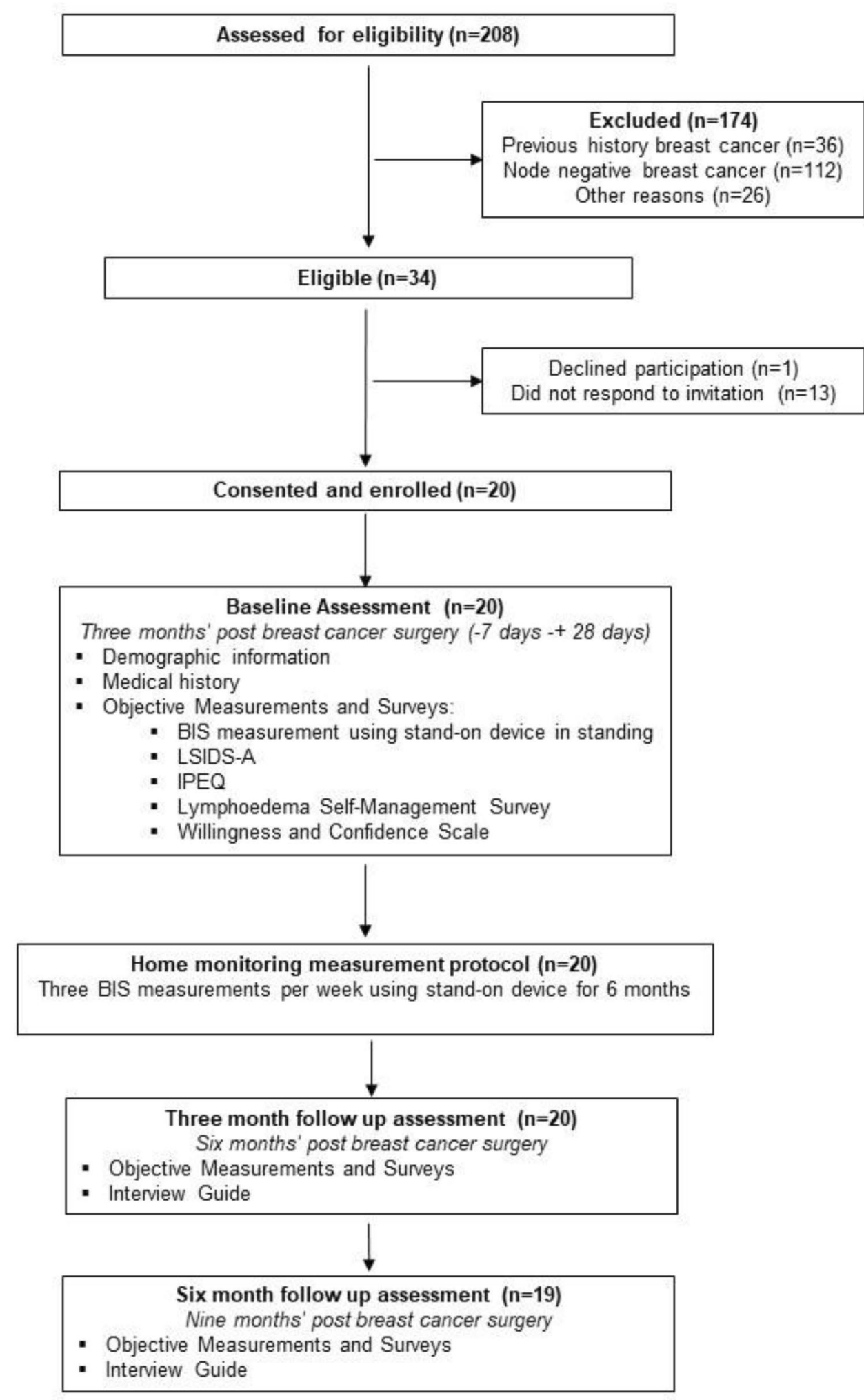

change of +6.5 L-Dex units from baseline is considered a trigger of sub-clinical lymphoedema [19, 29]. This device is portable and can be operated easily by an individual completing self-measurements within a home environment with access to power and a wireless internet network. The standon device was set up within the home (bedroom, bathroom, study or living area) and training was provided on how to take measurements and how to record and interpret results. 
Participants were advised to take the measurement three times a week, in the morning after waking and voiding in line with the frequency used in the previous pilot study [23]. To facilitate understanding of their own condition, participants were asked to record their L-Dex measurement and relevant comments (e.g. weather changes, activity, air travel, changes in treatment) on the home measurement log form.

Participants were advised to contact the research team if their L-Dex score increased by +6.5 points or more (defined as sub-clinical lymphoedema) from baseline result or if they had any lymphoedema-related symptoms or concerns. The device was controlled with an Android tablet pre-installed with the SOZOapp (ImpediMed Limited, Brisbane, Australia). All user accounts, measurement data, and other calculated measures and trends were stored in the secure MySOZO cloud (ImpediMed Limited, Brisbane, Australia) and BIS measurements were monitored remotely by the research team through this secure connection. Research personnel contacted participants by telephone or email if their L-Dex score increased above normal or if they observed any clinical or technical issues relating to lymphoedema or the study. At any time, participants who had an increase in extracellular fluid or lymphoedema symptoms were referred to a qualified lymphoedema therapist for standard clinical care, and this did not affect their ability to remain on study.

\section{Outcome measures}

\section{Feasibility}

Feasibility of recruitment, intervention and measurement was determined by examining the eligible, enrolled and retained participants as a proportion of individuals diagnosed with breast cancer from the three multidisciplinary breast cancer clinics. Feasibility of the intervention was defined as adherence, confidence in completing the intervention, usefulness of BIS home monitoring and number of technical issues encountered. Adherence was determined by recording the number of BIS measurements taken by participants according to the three times-per-week protocol and expressed as a percentage. Confidence was determined by the Willingness and Confidence Scale (adapted from Ridner et al. [25]) —a 10-item scale where confidence to incorporate self-monitoring into a self-management program was rated from 1 to 5 (strongly agree to strongly disagree) and reported as a score out of 50. Usefulness was determined by responses to questions about the advantages and disadvantages of use of the device, and the likelihood of using and/or purchasing the device. Technical issues were determined by counting the number of issues encountered using the standon device. Feasibility of measurement was defined as distance travelled to collect measures, duration and proportion of clinical outcomes completed.

\section{Clinical outcomes}

The primary clinical outcomes were (i) lymphoedema measured by BIS and reported as L-Dex scores taken from secure remote database and (ii) the proportion of participants whose L-Dex score increased by +6.5 or more from baseline. Secondary clinical outcomes included self-reported symptoms and distress, number of self-management strategies used and amount of physical activity. Symptoms and distress were measured using the Lymphoedema Symptom Intensity and Distress Survey-Arm Version 2.0 (LSIDS-A) [30]. This standardised and validated tool assessed 30 lymphoedema-related symptoms over the previous week. Intensity of symptoms and level of distress were rated on a 5-point Likert scale. Selfmanagement strategies were measured as the number of strategies (skin care, exercise, self-lymphatic drainage massage, compression, pneumatic compression pump) used over the previous 3 months. Physical activity was measured in terms of total and planned activity using the Incidental and Planned Exercise Questionnaire (IPEQ) [31, 32]. This standardised and validated tool comprises 12 questions about what physical activity and exercise has been done over the previous week and is reported in hours per week. The tool assesses low, basic and high intensity activities and was originally aimed for use in ageing research including fall prevention trials [32]. There are two levels of physical activity, planned physical activities that focus on planned exercise and planned walks and incidental physical activities that focus on more casual day-to-day activities [32].

\section{Data analysis}

Feasibility and participant characteristics are presented as mean (SD) and number (\%). All data were analysed using the IBM SPSS Statistics software package version 26 . For feasibility and clinical outcomes, either paired $t$-tests or the non-parametric equivalent Wilcoxon signed ranks test were used, depending on test assumptions and level of measurement, to examine between-time differences, between baseline and 3 months to evaluate engagement in home monitoring and self-management, and between baseline and 6 months to assess implementation of home monitoring and self-management. The tests yielded an identical pattern of statistical significance except in one instance indicated in Table 2, so for ease of clinical interpretation the data are presented as mean differences with $95 \%$ confidence intervals. 


\section{Results}

\section{Flow of participants through the study}

Twenty women of average age 54 (SD 12) years and mostly married and in paid employment participated in the study. Most participants had undergone an axillary lymph node dissection (95\%), completed radiotherapy and chemotherapy including taxane therapies $(90 \%)$ and were at high risk for developing unilateral arm lymphoedema (Table 1). The flow of participants through the study is shown in Fig. 1. One participant withdrew before the final measurement.

Table 1 Baseline characteristics of participants

\begin{tabular}{|c|c|}
\hline Characteristic & $N=20$ \\
\hline Age (years), mean (SD, range) & $54(12,35-78)$ \\
\hline BMI (kg), mean (SD, range) & $26(5,18-39)$ \\
\hline Arm at risk, $n$ right (\%) & $12(60)$ \\
\hline Hand dominance, $n$ right (\%) & $18(90)$ \\
\hline Marital status, $n$ married (\%) & $18(90)$ \\
\hline \multicolumn{2}{|l|}{ Highest level of education, $\mathrm{n}(\%)$} \\
\hline$<$ Year 10 & $3(15)$ \\
\hline High school certificate & $2(10)$ \\
\hline Vocational/TAFE & $3(15)$ \\
\hline Undergraduate degree & $6(30)$ \\
\hline Postgraduate degree & $6(30)$ \\
\hline \multicolumn{2}{|l|}{ Household income (\$AUD), $n(\%)$} \\
\hline$<\$ 50,000$ & $1(5)$ \\
\hline$\$ 50,000$ to $\$ 99,000$ & $1(5)$ \\
\hline$\$ 100,000$ to $\$ 150,000$ & $2(10)$ \\
\hline$>\$ 150,000$ & $6(30)$ \\
\hline Prefer not to say & $10(50)$ \\
\hline \multicolumn{2}{|l|}{ Paid Employment, $n(\%)$} \\
\hline Full-time & $6(30)$ \\
\hline Part-time & $6(30)$ \\
\hline Retired & $3(15)$ \\
\hline On leave (breast cancer treatment) & $2(10)$ \\
\hline Home duties & $3(15)$ \\
\hline Surgery type, $n$ mastectomy (\%) & $14(70)$ \\
\hline Node dissection, $n$ ALND (\%) & $19(95)$ \\
\hline \multicolumn{2}{|l|}{ Medical intervention, $n(\%)$} \\
\hline $\mathrm{RT}+\mathrm{NACT}\left(\right.$ with taxane) ${ }^{\mathrm{a}}$ & $7(35)$ \\
\hline $\mathrm{RT}+\mathrm{NACT}\left(\right.$ without taxane) ${ }^{\mathrm{a}}$ & $1(5)$ \\
\hline $\mathrm{RT}+\mathrm{CT}\left(\right.$ with taxane) ${ }^{\mathrm{a}}$ & $11(55)$ \\
\hline $\mathrm{RT}+\mathrm{CT}\left(\right.$ without taxane) ${ }^{\mathrm{a}}$ & $1(5)$ \\
\hline
\end{tabular}

$R T$ radiotherapy, $C T$ chemotherapy, NACT neoadjuvant chemotherapy, $A L N D$ axillary lymph node dissection, $n$ number, $S D$ standard deviation, $k g$ kilograms, $\$ A U D$ Australian dollars

${ }^{a}$ Specific data on radiation fields were not available from clinical files

\section{Feasibility}

\section{Recruitment}

A total of 208 women following breast cancer surgery were screened for eligibility between July 2018 and September 2019. Of the 34 participants who were eligible, 20 (59\%) enrolled in the study. They were all encouraged to participate in the study by either their breast surgeon (35\%), medical oncologist (35\%), lymphoedema therapist $(25 \%)$ or chemotherapy nurse (5\%). One participant $(5 \%)$ withdrew from the study just prior to the 6-month measurement due to COVID-19.

\section{Adherence}

Participants adhered to the BIS monitoring on average $74 \%$ (SD 24; Range 33 to 117) of the time during the first 3 months which is equivalent to 2.2 measures per week. Adherence remained stable over the second 3-month period with an average of 74\% (SD 42; Range 8 to 194). Twenty percent of the participants who had no significant change in L-Dex scores in the initial 3-month period adhered on average $33 \%$ of the time (equivalent to less than one measure per week) in the second 3-month monitoring period. Likewise, $25 \%$ of participants who had a significant increase $(>+6.5)$ in L-Dex scores increased adherence to an average of $109 \%$ of the time (equivalent to 3.3 measures per week) in the second 3-month monitoring period.

\section{Confidence}

Following education, demonstration and training of BIS at baseline, the mean confidence score was 48.0 out of 50 (SD 4), (range 40.0-50.0). This indicated that they felt extremely confident to set up the device, take a measurement, record the measurement on a log sheet and contact the research study team as needed over the next 6 months. Confidence remained high at 3 and 6 months (mean 48.8, SD 3).

\section{Usefulness of BIS monitoring}

Participants reported advantages and disadvantages of using the stand-on BIS device for monitoring over 6 months. Advantages reported in a free text response included the ease of use in operating the stand-on BIS device and being able to self-measure at home; the usefulness of being able to self-monitor to identify trends and early identification of changes or problems; having "peace of mind, reassurance and confidence" that they could identify sub-clinical changes and implement early intervention; and having the research support team for education, advice and assistance, as well as knowing that the research team were also remotely 
monitoring the L-Dex scores to identify any changes or need for early intervention.

Disadvantages of using the stand-on BIS device for monitoring change between the 3 - and 6-month measurements. At the 3 month measurement technical issues including $\mathrm{WiFi}$ and self-test issues were frustrating; fluctuating or increasing L-Dex readings with self-monitoring was concerning; the size of the device being positioned in a set place within the home was concerning and there were challenges associated with getting into a routine of regularly taking the measurement protocol. At the 6-month assessment, the size and positioning of the device in the home was the most frustrating disadvantage followed by the technical issues and concerns regarding fluctuating readings and time taken to complete measurements to a lesser extent.

When participants were asked whether they would purchase a stand-on BIS device for home monitoring, 75\% agreed that they would purchase or rent a device if it was affordable, clinically indicated or they could receive financial support from government assistance or their private health insurance provider for the recommended monitoring period. Over $50 \%$ of the women reported that they would be happy to continue taking measurements three times a week or as recommended by their lymphoedema therapist.

\section{Technical issues}

Eighty-five percent reported a technical issue in the initial 3 months, although the mean number of issues per participant was only 2.6 (SD 2.4). Most issues related to self-testing of device, WiFi connection issues or the interpretation of the Cole-plots indicating that better hand/foot contact was needed on the electrodes to ensure a high-quality reading. Further training and education to participants on the importance of good contact between the skin and electrodes reduced this error. The number of technical issues reported reduced significantly by two issues per participant (95\% CI 0.7 to 3.2) during the second 3 months so that by 6 months only $35 \%$ of participants experienced technical issues with the mean number of issues per participant only 0.6 (SD 0.9).

\section{Measurement}

Clinical measures were collected for all participants at baseline and 3 months and for 19 participants at 6 months in the home. The mean distance travelled to collect measures was $26 \mathrm{~km}$ (SD 30) and the mean time taken to complete measurements and ongoing education and support components of the model of care was $99 \mathrm{~min}$ (SD 17) at baseline and $44 \mathrm{~min}$ (SD 12) for other measurements. Some women had many questions for the research team about lymphoedema, the monitoring program and generally how they were coping physically and psychologically through their medical treatment which sometimes varied or prolonged the study assessment time. The symptom and distress data are incomplete for five participants due to an error in initial setup of the electronic data collection system.

\section{Clinical outcomes}

Clinical outcomes are presented in Table 2. Over the 6-month period, from 3 to 9 months after surgery there was a general increase in L-Dex scores. Five women (25\%) experienced increased extracellular fluid levels with an L-Dex score $>+6.5$ from baseline. The mean time for triggering the $+6.5 \mathrm{~L}-$ Dex increase for these five women was 36 days (SD 30; Range 2-67) from baseline. Women were educated to contact the research team if their L-Dex reading increased $>+6.5$ from baseline. When this occurred, they were encouraged to contact their local qualified lymphoedema therapist for early intervention. This early intervention was not standardised for all women depending on the local lymphoedema therapist they consulted. Each of the five women were fitted with a compression garment and prescribed a self-management program. Self-reported symptoms and distress decreased by 0.4 out of 10.0 (95\% CI 0.1

Table 2 Mean (SD) outcomes over time and mean (95\% CI) difference between times

\begin{tabular}{|c|c|c|c|c|c|}
\hline \multirow[t]{2}{*}{ Outcome } & \multicolumn{3}{|l|}{ Times } & \multicolumn{2}{|l|}{ Difference over time } \\
\hline & Baseline $n=20$ & 3 months $n=20$ & 6 months $n=19$ & 3 months minus baseline & 6 months minus baseline \\
\hline Lymphoedema, BIS (L-Dex units) & $3.5(5.6)$ & $6.0(9.6)$ & $8.4(11.1)$ & $2.5(-1.1$ to 6.2$)$ & $4.8(-0.5$ to 10.2$)$ \\
\hline $\begin{array}{l}\text { Self-reported symptom and distress } \\
(0-10)\end{array}$ & $1.6(1.2) n=19$ & $1.1(0.9) n=19$ & $0.9(0.8) n=15$ & $-0.3(-0.6 \text { to }-0.0)^{* *}$ & $-0.4(-0.7 \text { to }-0.1)^{*}$ \\
\hline Self-management strategies $(0-5)$ & $1.9(1.1)$ & $2.1(1.0)$ & $2.6(1.2)$ & $0.3(-0.4$ to 0.9$)$ & $0.6(0.0 \text { to } 1.2)^{*}$ \\
\hline \multicolumn{6}{|l|}{ Physical activity (h/week) } \\
\hline Total (incidental + planned) & $36(13)$ & $38(24)$ & $33(15)$ & $2(-5$ to 10$)$ & $-3(-11$ to 4$)$ \\
\hline Planned & $3.5(3.2)$ & $5.6(7.8)$ & $5.9(5.1)$ & $2.0(-1.5$ to 5.5$)$ & $2.8(0.4 \text { to } 5.2)^{*}$ \\
\hline
\end{tabular}

BIS bioimpedance spectroscopy

$* p<0.05 ; * *<0.04$ for paired T-test but Wilcoxon signed ranks test $p=0.052$ 
to 0.7$)$ over 6 months. The number of self-management strategies increased by 0.6 per participant $(95 \%$ CI 0.1 to 1.2$)$ over 6 months. Overall, there was no increase in total (incidental + planned) physical activity but there was an increase of $2.8 \mathrm{~h} /$ week (95\% CI 0.4 to 5.2) of planned exercise over 6 months (Table 2).

\section{Discussion}

A prospective surveillance model of care delivered in the home consisting of BIS monitoring, education, and support to promote self-management and physical activity for women at high risk of developing lymphoedema appears to be feasible in terms of recruitment, intervention and measurement. Only one participant withdrew from the study and the remaining participants reported decreasing lymphoedema symptom intensity and distress, increasing lymphoedema self-management, and increased planned physical activity over 6 months. Total activity and exercise completed should be interpreted with caution as $35 \%$ of participants were completing home monitoring during the COVID-19 period when isolation and lock-down periods were implemented.

The use of home monitoring and self-management interventions are having positive impacts in several health conditions [33-35]. With 59\% recruitment of eligible women to the study, it seems that women who are at high risk for developing lymphoedema are also interested and committed to being involved in whatever they can do to reduce their risk. These women had been told by their multidisciplinary breast cancer team that they were at high risk of developing lymphoedema and were recommended to participate in the study. The concept of a home program was appealing to these women who reported that it "empowered them" and "gave them some control" as many reported feeling "out of control" and "overwhelmed" at the time of recruitment to the study. Furthermore, the $95 \%$ retention rate suggests that the women were engaged in the program.

Women generally adhered to BIS monitoring three times per week. Those who had stable L-Dex scores in the initial 3-month monitoring period decreased the frequency of BIS measurements during the second 3-month monitoring period. Likewise, those who had increasing L-Dex scores increased the frequency of measurements during the second 3-month monitoring period. Participants in a qualitative study exploring the concept of home monitoring using BIS reported that receiving objective measurements via BIS home monitoring may positively affect adherence to self-management, but that it was important to have clear guidelines for how to action any elevations or fluctuations in readings. It was agreed by participants in these focus groups that the frequency of home measurements should differ, with those at risk and/or in early stages of sub-clinical lymphoedema enacting more frequent measurements than those with more stable and advanced clinical lymphoedema [24]. This may reflect the benefit of taking objective measurements to change behaviour and adherence to self-management [23]. Our findings of extremely high levels of confidence in BIS monitoring following appropriate training and support are in line with a previous pilot study [25]. Importantly, technical issues reduced over the 6 months. Technical issues experienced in the early stages of the study period highlighted the need for ongoing education and support to individuals to understand the specific aspects of correct setup, internet connectivity and reviewing of Cole-plots. This aspect of the measurement process has subsequently been improved by an enhancement to the device software with the addition of a traffic light colour coding system (green, amber and red) to assist women in only accepting good quality measurements. There was also a typical learning curve for the research study team as they became more familiar with the new technology over time and their problem solving skills, education and advice they were able to give to participants ensured more accurate taking of measurements.

It appears that the prospective surveillance model of care delivered in the home has the potential to be beneficial. Ideally women would be introduced to a prospective surveillance model of care at the time of their breast cancer diagnosis within the hospital or clinic setting as routine practice and then introduced to additional home monitoring at a time following the initial stress of diagnosis had settled and they were able to take on further information. Although BIS L-Dex scores increased, only five participants in this group of high-risk women met the criteria for sub-clinical lymphoedema and many of these were still undergoing adjuvant chemotherapy or radiotherapy treatment during this time. Interestingly, in these participants the time to + 6.5 L-Dex score was quite variable (2 days to 67 days) which suggests adherence to home monitoring is important for timely intervention. This cohort of women were 3 months post-surgery for breast cancer and reported low levels of symptoms causing distress. The symptoms they did report may have still been associated with the physical recovery of the surgery and associated biomechanical and musculo-skeletal issues [36]. The women increased the number of self-management strategies that they implemented over the 6 months, with the most notable increases being in exercise and use 
of compression. Skin care was the most utilised strategy with $>85 \%$ undertaking it at baseline [37-40]. Planned physical activity increased over 6 months to 5.9 h/week, a frequency that exceeded the recommended Clinical Society of Oncology guidelines of $2.5 \mathrm{~h} /$ week [41]. However, we do not know about the intensity of the planned exercise. International guidelines recommend that those diagnosed with cancer should progress towards and maintain participation in at least $150 \mathrm{~min}$ of moderate intensity or $75 \mathrm{~min}$ of vigorous-intensity aerobic exercise (e.g. walking, jogging, cycling, swimming) each week; and two to three resistance exercise (i.e. lifting weights) sessions each week involving moderate to vigorous-intensity exercises targeting the major muscle groups [41, 42].

\section{Conclusion}

This Phase I feasibility study suggests that a prospective surveillance model of care delivered in the home consisting of BIS monitoring, education and support to promote self-management and physical activity for women at high risk of developing lymphoedema is feasible and has the potential to be beneficial. At a time when individuals often have their diaries filled with medical, allied health and treatment appointments, BIS home monitoring may support and empower individuals to reduce unnecessary appointments and trips to hospitals and clinics if they know that their fluid levels are not fluctuating and are feeling confident in the prospective surveillance model at home. These findings suggest that a Phase II randomised trial of longer duration given that these women are at high risk of developing lymphoedema for up to 3 years $[13,14]$ is warranted. In addition, research exploring the variations in L-Dex readings over time, assessing anxiety and distress levels and costs associated with implementing this model of care for high-risk individuals is necessary.

Acknowledgements Louise Ada for editorial assistance.
Author contributions LAK: conceptualisation, data collection and curation, formal analysis, funding acquisition, investigation, methodology, project administration, resources, writing—original draft, and writing - review and editing. EM: data collection, data analysis, writing - review and editing. JB: conceptualisation, supervision, writing-review and editing. KAS: conceptualisation, supervision, writing-review and editing. CD: conceptualisation, data curation and analysis, methodology, supervision, writing — original draft, and writing-review and editing.

Funding This research was financially supported by a Grant from ImpediMed Limited to the Australian Lymphoedema Education, Research and Treatment (ALERT) Program, Macquarie University for data collection. ImpediMed Limited supplied the use of $20 \mathrm{SOZO}$ Bioimpedance Spectroscopy devices which were loaned to women participating in this study. Devices will be returned to ImpediMed Limited at the conclusion of the study. Koelmeyer was awarded a Sydney Vital Research grant for \$10000AUD which was used towards travelling costs to participants' homes, purchase of electronic scales and presenting study results at relevant conferences.

\section{Compliance with ethical standards}

Conflict of interest This research was financially supported by a grant from ImpediMed Limited to the Australian Lymphoedema Education, Research and Treatment (ALERT) Program, Macquarie University for data collection. Author Koelmeyer has acted as an Education Consultant to ImpediMed Limited. Boyages is a shareholder in ImpediMed. Authors Dean and Sherman declare that they have no conflicts of interest or financial ties to disclose. ImpediMed Limited was not involved in the conception, design, execution, data analysis for this study or in the preparation of the manuscript. ImpediMed Limited was sent the final draft version prior to submission to confirm technical information was accurate.

Ethical approval Macquarie University Human Research Ethics Committee, Reference No: 5201800197 and Adventist HealthCare Limited Human Research Ethics Committee Reference number 2018-036.

\section{Appendix}

See Table 3. 
Table 3 TIDIER checklist

TIDieR criteria

Item 1: Brief name or phrase that describes the intervention

Item 2: Rationale, theory, or goal of the elements essential to the intervention

Item 3: What (Materials): any physical or informational materials used in the intervention, including those provided to participants or used in intervention delivery or in training of intervention providers
Experimental intervention

Prospective surveillance model of care in the home

A prospective surveillance and early intervention model of care has been recognised as an optimal framework for the early detection and management of sub-clinical lymphoedema [13-15]. Using prospective surveillance model in clinical practice enables the early identification and intervention for lymphoedema, sometimes in a sub-clinical stage, where intervention can prevent progression to a chronic condition [16-19]. Recommended practice for the prospective surveillance model requires that individuals at risk of lymphoedema regularly attend clinics on a 3-6 monthly cycle according to their risk factors and treatment pathway for three years to have their arm monitored for lymphoedema by either using bioimpedance spectroscopy (BIS) or circumference measurements $[13,16]$

This study assessed the feasibility of delivering a prospective surveillance model of care in the home to participants who were aged between 18 and 85 years with a histologically confirmed node-positive invasive breast cancer after undergoing an axillary lymph node dissection or sentinel lymph node biopsy, were able to speak and read English and were capable of giving informed written consent participated in the study. They were excluded if they had implantable devices such as pacemaker or other inbuilt stimulator, were pregnant, reported having a previous history of breast cancer or arm lymphoedema, if they had a health condition that may affect body fluid status or if they reported having a psychiatric illness that would limit their compliance with study requirements

The intervention consisted of a package including BIS monitoring, education, and support to promote self-management and physical activity over a 6-month period

BIS monitoring involved the use of a BIS stand-on device. The stand-on device used was a commercially available impedance device (SOZOß, ImpediMed Limited, Brisbane, Australia) with demonstrated validity and reliability [28]. This device is portable and can be operated easily by an individual completing self-measurements within a home environment with access to power and a wireless internet network

Electronic scales were used so that the participant could take their weight prior to a BIS measurement

The education component focussed on risk minimisation and included provision of the Australian Cancer Council Understanding Lymphoedema fact sheet [27]

Self-management strategies included skin care, exercise, self-lymphatic drainage massage, compression and the use of pneumatic compression. Information about these strategies was provided to participants by research team and included in the Australian Cancer Council Understanding Lymphoedema fact sheet [27]

To facilitate understanding of their own condition, participants were asked to record their L-Dex measurement and relevant comments (e.g. weather changes, activity, air travel, changes in treatment) in the home measurement $\log$ form 
Table 3 (continued)

\section{TIDieR criteria}

Item 4: What (Procedures): the procedures, activities, and/or processes used in the intervention, including any enabling or support activities

Item 5: Who provided, their expertise, background and any specific training given

Item 6: Modes of delivery (e.g. Face-to-face or by some other mechanism, such as internet or telephone) of the intervention and whether it was provided individually or in a group

Item 7: Where, the type(s) of location(s) where the intervention occurred, including any necessary infrastructure or relevant features

Item 8: When and how much. A description of the number of times the intervention was delivered and over what period of time including the number of sessions, their schedule, and their duration, intensity or dose

Item 9: Tailoring, if the intervention was planned to be personalised, titrated or adapted, then describe what, why, when, and how

Item 10: Modifications. If the intervention was modified during the course of the study, describe the changes (what, why, when, and how)
Experimental intervention

Women were screened via the telephone and eligible participants were scheduled for a baseline assessment in their home 3 months after breast cancer surgery. Measurements were taken at baseline, 3 and 6 months in the home by trained measurers following standardised protocols. Intervention was provided at the conclusion of each measurement session

The stand-on BIS device was set up within the home (bedroom, bathroom, study or living area) and training was provided of how to take measurements and how to record and interpret results. Participants were advised to take the measurement three times a week, in the morning after waking and voiding. Participants were advised to contact research team if their L-Dex score increased by $>+6.5$ points or more (defined as sub-clinical lymphoedema) from baseline result or if they had any lymphoedema-related symptoms or concerns The device is controlled with an Android tablet pre-installed with the SOZOapp (ImpediMed Limited, Brisbane, Australia). All user accounts, measurement data, and other calculated measures and trends were stored in the secure MySOZO cloud (ImpediMed Limited, Brisbane, Australia) and BIS measurements were monitored remotely by the research team through this secure connection

Research personnel contacted participants by telephone or email if their L-Dex score increased above normal or if they observed any clinical or technical issues relating to lymphoedema or the study. At any time, participants who had an increase in extracellular fluid or lymphoedema symptoms were referred to a qualified lymphoedema therapist for standard clinical care, and this did not affect their ability to remain on study

The prospective surveillance model of care in the home was delivered by a qualified occupational and lymphoedema therapist, registered with the appropriate professional body with support for data collection and liaison with participants by a trained research assistant

The prospective surveillance model of care in the home was provided individually:

Face-to-face provision of the intervention program

Telephone calls and email communication to participants for any study related or technical support

The prospective surveillance model of care was delivered in the home environment

The BIS stand-on device and electronic scales were loaned to the participant for the duration of the study and wipes for infection control and dampening the electrodes were supplied by study team

The prospective surveillance model of care was standardised to a 6-month program. Participants were advised to take the BIS measurement three times a week, in the morning after waking and voiding Measurements were taken at baseline, 3 and 6 months in the home by trained measurers following standardised protocols

The prospective surveillance model of care was standardised; however at any time, participants who had an increase in extracellular fluid or lymphoedema symptoms were referred to a qualified lymphoedema therapist for standard clinical care, and this did not affect their ability to remain on study

No modifications were made to the standardised protocol after participant recruitment began. Further training and education to participants on the importance of good contact between the skin and electrodes reduced further technical errors when using the stand-on device to take an accurate measurement 
Table 3 (continued)

\section{TIDieR criteria}

Item 11: How well (Planned) If intervention adherence or fidelity was assessed, describe how and by whom, and if any strategies were used to maintain or improve fidelity, describe them

Item 12: How well (Actual) If intervention adherence or fidelity was assessed, describe the extent to which the intervention was delivered as planned
Experimental intervention

Audit of documentation to assess provision of the prospective surveil-

lance model of care within the home

Participant recorded details of the number of BIS measurements taken in the home measurement log form

Double entry and external checking of all data inputted into electronic database

To facilitate understanding of their own condition and participation in the prospective surveillance model of care, participants were asked to record their L-Dex measurement and relevant comments (e.g. weather changes, activity, air travel, changes in treatment) in the home measurement $\log$ form

\section{References}

1. Hormes JM, Bryan C, Lytle LA, Gross CR, Ahmed RL, Troxel AB, Schmitz KH (2010) Impact of lymphedema and arm symptoms on quality of life in breast cancer survivors. Lymphology 43(1):1-13

2. Armer JM (2005) The problem of post-breast cancer lymphedema: impact and measurement issues. Cancer Investig 23(1):76-83

3. Hayes S, Di Sipio T, Rye S, Lopez JA, Saunders C, Pyke C, Bashford J, Battistutta D, Newman B (2011) Prevalence and prognostic significance of secondary lymphedema following breast cancer. Lymphat Res Biol 9(3):135-141

4. DiSipio T, Rye S, Newman B, Hayes S (2013) Incidence of unilateral arm lymphoedema after breast cancer: a systematic review and meta-analysis. Lancet Oncol 14(6):500-515. https://doi. org/10.1016/s1470-2045(13)70076-7

5. Hayes SC, Janda M, Cornish B, Battistutta D, Newman B (2008) Lymphedema after breast cancer: incidence, risk factors, and effect on upper body function. J Clin Oncol 26(21):3536-3542

6. Perdomo M, Davies C, Levenhagen K, Ryans K (2014) Breast cancer edge task force outcomes: assessment measures of secondary lymphedema in breast cancer survivors. Rehabil Oncol 32(1):22-35

7. Cormier JN, Askew RL, Mungovan KS, Xing Y, Ross MI, Armer JM (2010) Lymphedema beyond breast cancer: a systematic review and meta-analysis of cancer-related secondary lymphedema. Cancer 116(22):5138-5149. https://doi. org/10.1002/cncr.25458

8. Armer J, Hulett J, Bernas M, Ostby P, Stewart B, Cormier J (2013) Best-practice guidelines in assessment, risk reduction, management, and surveillance for post-breast cancer lymphedema. Current Breast Cancer Reports 5(2):134-144. https://doi.org/10.1007/ s12609-013-0105-0

9. Ancukiewicz M, Russell TA, Otoole J, Specht M, Singer M, Kelada A, Murphy CD, Pogachar J, Gioioso V, Patel M, Skolny M, Smith BL, Taghian AG (2011) Standardized method for quantification of developing lymphedema in patients treated for breast cancer. Int J Radiat Oncol Biol Phys 79(5):1436-1443. https://doi. org/10.1016/j.ijrobp.2010.01.001

10. Boyages J, Xu Y, Kalfa S, Koelmeyer L, Parkinson B, Mackie H, Viveros H, Gollan P, Taksa L (2016) Financial cost of lymphedema borne by women with breast cancer. Psycho-oncology 26(6):849-855. https://doi.org/10.1002/pon.4239

11. Wetzig N, Gill PG, Zannino D, Stockler MR, Gebski V, Ung O, Campbell I, Simes RJ (2014) Sentinel lymph node based management or routine axillary clearance? Three-year outcomes of the RACS sentinel node biopsy versus axillary clearance (SNAC) 1 trial. Ann Surg Oncol. https://doi.org/10.1245/s10434-014-3928-7
12. Stout Gergich NL, Pfalzer LA, McGarvey C, Springer B, Gerber LH, Soballe P (2008) Preoperative assessment enables the early diagnosis and successful treatment of lymphedema. Cancer 112(12):2809-2819

13. McLaughlin SA, Stout NL, Schaverien MV (2020) Avoiding the swell: advances in lymphedema prevention, detection, and management. Am Soc Clin Oncol. https://doi.org/10.1200/ EDBK_280471

14. Dylke ES (2019) Position paper: early detection of breast cancerrelated lymphoedema. Australasian Lymphology Association; https://www.lymphoedemaorgau/public/7/files/Position\%2520S tatements/ALA\%2520Position\%2520Statement_Early\%2520D etection\%2520of\%2520BCRLpdf. Accessed 26 July 2020

15. Shah C, Arthur DW, Wazer D, Khan A, Ridner S, Vicini F (2016) The impact of early detection and intervention of breast cancerrelated lymphedema: a systematic review. Cancer Med 5(6):1154 1162. https://doi.org/10.1002/cam4.691

16. Koelmeyer LA, Borotkanics RJ, Alcorso J, Prah P, Winch CJ, Nakhel K, Dean CM, Boyages J (2019) Early surveillance is associated with less incidence and severity of breast cancer-related lymphedema compared with a traditional referral model of care. Cancer 125(6):854-862. https://doi.org/10.1002/cncr.31873

17. Kilgore LJ, Korentager SS, Hangge AN, Amin AL, Balanoff CR, Larson KE, Mitchell MP, Chen JG, Burgen E, Khan QJ, O'Dea AP, Nye L, Sharma P, Wagner JL (2018) Reducing breast cancerrelated lymphedema (BCRL) through prospective surveillance monitoring using bioimpedance spectroscopy (BIS) and patient directed self-interventions. Ann Surg Oncol 25(10):2948-2952. https://doi.org/10.1245/s10434-018-6601-8

18. Whitworth PW, Shah C, Vicini F, Cooper A (2018) Preventing breast cancer-related lymphedema in high-risk patients: the impact of a structured surveillance protocol using bioimpedance spectroscopy. Front Oncol 8:197. https://doi.org/10.3389/fonc.2018.00197

19. Ridner SH, Dietrich MS, Cowher MS, Taback B, McLaughlin S, Ajkay N, Boyages J, Koelmeyer L, DeSnyder SM, Wagner J, Abramson V, Moore A, Shah S (2019) A randomized trial evaluating bioimpedance spectroscopy versus tape measurement for the prevention of lymphedema following treatment for breast cancer: interim analysis. Ann Surg Oncol 26:3250-3259. https://doi. org/10.1245/s10434-019-07344-5

20. Ward LC (2006) Bioelectrical impedance analysis: proven utility in lymphedema risk assessment and therapeutic monitoring. Lymphat Res Biol 4(1):51-56

21. Soran A, Ozmen T, McGuire KP, Diego EJ, McAuliffe PF, Bonaventura M, Ahrendt GM, DeGore L, Johnson R (2014) The importance of detection of subclinical lymphedema for the prevention of breast cancer-related clinical lymphedema after axillary lymph node dissection; a prospective observational study. 
Lymphat Res Biol 12(4):289-294. https://doi.org/10.1089/ lrb.2014.0035

22. Ward LC (2015) Bioelectrical impedance spectrometry for the assessment of lymphoedema: principles and practice. In: Greene AK, Slavin SA, Brorson H (eds) Lymphedema. Springer, Cham, pp 123-132

23. Ridner SH, Shih YC, Doersam JK, Rhoten BA, Schultze BS, Dietrich MS (2014) A pilot randomized trial evaluating lymphedema self-measurement with bioelectrical impedance, self-care adherence, and health outcomes. Lymphat Res Biol 12(4):258-266. https://doi.org/10.1089/lrb.2014.0017

24. Koelmeyer L, Sherman KA, Boyages J (2018) Understanding self-management and home monitoring in breast cancer-related lymphoedema: a qualitative study. Paper presented at the 12th Australasian Lymphology Association Conference, Brisbane, QLD, Australia, 17-19 May 2018

25. Ridner SH, Bonner CM, Doersam JK, Rhoten BA, Schultze B, Dietrich MS (2014) Bioelectrical impedance self-measurement protocol development and daily variation between healthy volunteers and breast cancer survivors with lymphedema. Lymphat Res Biol. https://doi.org/10.1089/lrb.2013.0020

26. Ridner SH, Dietrich MS, Kidd N (2011) Breast cancer treatment-related lymphedema self-care: education, practices, symptoms, and quality of life. Support Care Cancer 19(5):631-637. https://doi.org/10.1007/s00520-010-0870-5

27. Cancer Council Australia (2017) Understanding lymphoedema: a guide for people affected by cancer. Brisbane, Australia

28. Koelmeyer LA, Ward LC, Dean C, Boyages J (2020) Body positional effects on bioimpedance spectroscopy measurements for lymphedema assessment of the arm. Lymphat Res Biol. https:// doi.org/10.1089/1rb.2019.0067

29. Fu MR, Cleland CM, Guth AA, Kayal M, Haber J, Cartwright F, Kleinman R, Kang Y, Scagliola J, Axelrod D (2013) L-dex ratio in detecting breast cancer-related lymphedema: reliability, sensitivity, and specificity. Lymphology 46(2):85-96

30. Ridner SH, Dietrich MS (2015) Development and validation of the lymphedema symptom and intensity survey-arm. Support Care Cancer 23(10):3103-3112. https://doi.org/10.1007/s0052 0-015-2684-y

31. Merom D, Delbaere K, Cumming R, Voukelatos A, Rissel C, Van Der Ploeg HP, Lord SR (2014) Incidental and planned exercise questionnaire for seniors: validity and responsiveness. Med Sci Sports Exerc 46(5):947-954. https://doi.org/10.1249/ MSS.0000000000000196

32. Delbaere K, Hauer K, Lord SR (2010) Evaluation of the incidental and planned exercise questionnaire (IPEQ) for older people. Br J Sports Med 44:1029-1034. https://doi.org/10.1136/ bjsm.2009.060350

33. Eguchi K, Hoshide S, Ishikawa S, Shimada K, Kario K (2012) Aggressive blood pressure-lowering therapy guided by home blood pressure monitoring improves target organ damage in hypertensive patients with type 2 diabetes/prediabetes. J Clin
Hypertens (Greenwich) 14(7):422-428. https://doi.org/10.111 1/j.1751-7176.2012.00648.x

34. Erie C, Van Name MA, Weyman K, Weinzimer SA, Finnegan J, Sikes K, Tamborlane WV, Sherr JL (2018) Schooling diabetes: use of continuous glucose monitoring and remote monitors in the home and school settings. Pediatr Diabetes 19(1):92-97. https:// doi.org/10.1111/pedi.12518

35. Basatneh R, Najafi B, Armstrong DG (2018) Health sensors, smart home devices, and the internet of medical things: an opportunity for dramatic improvement in care for the lower extremity complications of diabetes. J Diabetes Sci Technol 12(3):577-586. https ://doi.org/10.1177/1932296818768618

36. Stout NL, Binkley JM, Schmitz KH, Andrews K, Hayes SC, Campbell KL, McNeely ML, Soballe PW, Berger AM, Cheville AL, Fabian C, Gerber LH, Harris SR, Johansson K, Pusic AL, Prosnitz RG, Smith RA (2012) A prospective surveillance model for rehabilitation for women with breast cancer. Cancer 118(8 Suppl):2191-2200. https://doi.org/10.1002/cncr.27476

37. Alcorso J, Sherman KA, Koelmeyer L, Mackie H, Boyages J (2016) Perceived barriers to adherence to breast cancer-related lymphoedema self-management. J Lymphoedema 11(1):20-26

38. Sherman KA, Miller SM, Roussi P, Taylor A (2015) Factors predicting adherence to risk management behaviors of women at increased risk for developing lymphedema. Support Care Cancer 23(1):61-69. https://doi.org/10.1007/s00520-014-2321-1

39. Sherman KA, Koelmeyer L (2013) Psychosocial predictors of adherence to lymphedema risk minimization guidelines among women with breast cancer. Psycho-oncology 22(5):1120-1126. https://doi.org/10.1002/pon.3111

40. Sherman KA, Koelmeyer L (2011) The role of information sources and objective risk status on lymphedema risk-minimization behaviors in women recently diagnosed with breast cancer. Oncol Nurs Forum 38(1):E27-36. https://doi.org/10.1188/11.onf. e27-e36

41. Clinical Oncological Society of Australia (2018) COSA position statement on exercise in cancer care. COSA, Sydney, Australia

42. Sanft T, Denlinger CS, Armenian S, Baker KS, Broderick G, Demark-Wahnefried W, Friedman DL, Goldman M, Hudson M, Khakpour N, Koura D, Lally RM, Langbaum TS, McDonough AL, Melisko M, Mooney K, Moore HCF, Moslehi JJ, O'Connor T, Overholser L, Paskett ED, Peterson L, Pirl W, Rodriguez MA, Ruddy KJ, Smith S, Syrjala KL, Tevaarwerk A, Urba SG, Zee P, McMillian NR, Freedman-Cass DA (2019) NCCN guidelines insights: survivorship version 2.2019. J Natl Compr Cancer Netw JNCCN 17(7):784-794. https://doi.org/10.6004/jnccn.2019.0034

Publisher's Note Springer Nature remains neutral with regard to jurisdictional claims in published maps and institutional affiliations. 